\title{
A review of the important central role of altered citrate metabolism during the process of stem cell differentiation
}

\author{
Leslie C. Costello* and Renty B. Franklin \\ *Correspondence: Icostello@umaryland.edu \\ Department of Oncology and Diagnostic Sciences, University of Maryland Dental School and The University of Maryland \\ Greenebaum Cancer Center, Baltimore, Maryland 21201, USA.
}

\begin{abstract}
Stem cells are highly proliferating cells that have the potential for differentiation leading to the development of specialized functional cell types. The process of stem cell differentiation requires an increase in the recruitment and population of the undifferentiated stem cells, which are then differentiated to specific functional cell types. Genetic/metabolic transformations in the cellular intermediary energy metabolism are required to provide the bioenergetic, synthetic, and catabolic requirements of the stem cells during this process. However, the identification of the intermediary energy metabolism pathways and their alterations during the proliferation and differentiation of stem cells remain largely unknown; mainly due to the lack of attention and/or required research that focuses on this relationship. In the absence of such information, a full understanding of the factors and conditions required to promote stem cell differentiation leading to development of normal functional metabolic specialized cells cannot be achieved. The purpose of this review is to provide the background and bring attention to the essential relationship of altered cellular intermediary metabolism in the context of the process of stem cell proliferation and differentiation. Citrate metabolism is central to the genetic and metabolic transformation leading to the development of the specialized functional cells. This review identifies the involvement of altered citrate metabolism and the associated genetic alterations of key pathways, enzymes, and transporters; as well as the bioenergetic implications. The importance is emphasized for identification and employment of required conditions to insure that the process of experimental stem cell differentiation results in the development of specialized cells that represent the functional metabolic characteristics and capabilities of their native specialized cells. This is an essential requirement for the successful application of stem cell therapy and regenerative medicine for many pathological conditions.
\end{abstract}

Keywords: Citrate metabolism, stem cells, differentiation and proliferation, osteogenesis, osteoblast and bone formation adipogenesis, krebs cycle, glycolysis

\section{Introduction}

The relationship between the activity of a cell and the metabolism of the cell is defined by the following indispensible axioms [1,2]: Axiom 1. The existing intermediary metabolism of a cell provides the bioenergetic/synthetic/catabolic requirements that are essential for the manifestation of the cell's current activity (eg., function, growth, proliferation, differentiation); Axiom 2. When the activity of a cell changes, its metabolism must also be altered to provide new bioenergetic/synthetic/catabolic requirements for the cell's changing activity. This relationship especially applies to stem cells during the process of differentiation, which includes: a) the increase in population of the undifferentiated stem cells; and b) their differentiation leading to specialized cell types that exhibit differing functional activities (Figure 1). The achievement of this process requires metabolic alterations in concert with the changing activity of the stem cells.

Consequently, the factors that promote and regulate the differentiation process must also include the regulation of the stem cell's metabolic pathways. This involves alterations of expression of "metabolic genes" associated with regulatory enzymes and transporters involved in the metabolic pathway; i.e., "genetic/metabolic" transformation is involved. A side from a few studies [such as 3-10] the metabolic implications in the differentiation process of stem cells have received little attention. In the absence of elucidation of the genetic/metabolic relationships and identification of the operation of specific metabolic pathways, an appropriate understanding of the process and factors involved in stem cell regeneration and differentiation cannot be achieved.

For the most part, stem cell biology and its clinical application is a relatively recent focus in biomedical and clinical research. Of the many issues that have been and are being addressed, the identification of stem cell intermediary metabolism pathways in relation to stem cell activities has received little attention and is largely unknown. The situation can be likened to that of tumor cell metabolism during the era of the "mitochondriacs" from 1940-1985; which was dominated by studies of pathways of intermediary energy metabolism in mammalian cells. That focus was applied to the developing interest in tumor cell metabolism; and gave rise to the marriage of the expertise in cell metabolism, biochemistry and enzymology with the metabolic implications in the development and progression of cancer. However, since $\sim 1985$, the advent and expansion of molecular biology,

C 2013 Costello et al; licensee Herbert Publications Ltd. This is an Open Access article distributed under the terms of Creative Commons Attribution License (http://creativecommons.org/licenses/by/3.0), This permits unrestricted use, distribution, and reproduction in any medium, provided the original work is properly cited. 

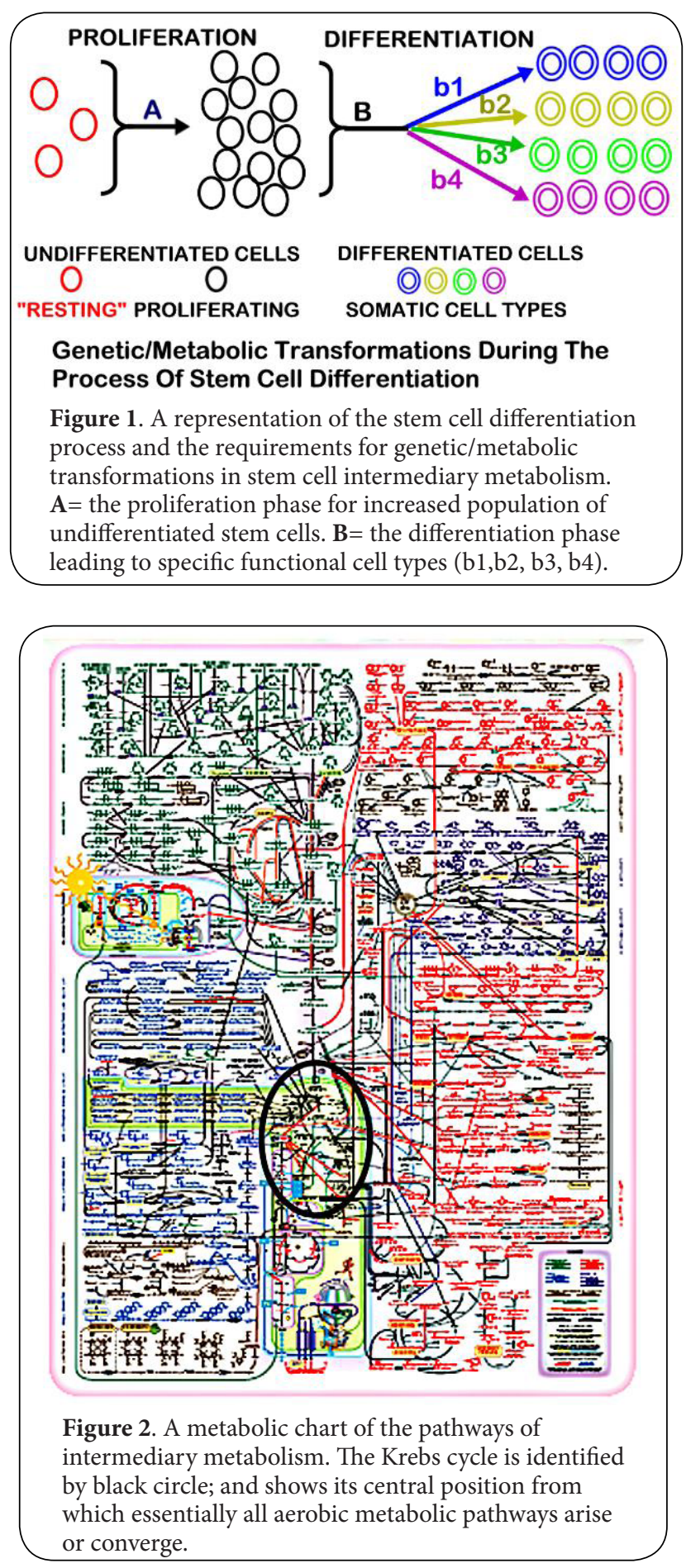

molecular genetics and genomics, proteomics. and molecular technology have dominated the biomedical and clinical interest. As a consequence, the focus, interest, and training in cell intermediary energy metabolism and biochemistry, enzymology, and metabolic methods greatly diminished. In more recent years, a renewed interest is evolving in the importance of cellular metabolism in the physiological and pathophysiological implications in medicine. This is evident from the current evolution of areas such as "metabolic medicine", "mitochondrial medicine", and "metabolomics". Thus a new marriage of cell metabolism and biochemistry with contemporary biomedical interests and issues relating to stem cell proliferation and differentiation should be implemented; as occurred decades ago for tumor cells and cancer. This will require intense interest and research, because there is no "quick and easy" appropriate way to identify operational cellular metabolic pathways and genetic/metabolic alterations. It also requires the knowledge and application of appropriate metabolic, biochemical and enzymological methods and approaches necessary to obtain accurate information and relevant translational interpretation. Hopefully, this review will provide a helpful background that will focus attention and expand the needed studies for elucidation of the metabolic implications in stem cells.

\section{Why focus on a central role of citrate metabolism in relation to stem cell differentiation?}

Fundamental and essential genetic/metabolic alterations occur when the undifferentiated "resting" stem cell is directed to highly active cell proliferation to increase its population; followed by genetic/metabolic transformations during the differentiation of the undifferentiated cells to specialized functional cell types. Essential to such metabolic transformations is the central role of altered citrate metabolism, which is the focus of this presentation. The role and pathways of altered citrate metabolism along with the key genetic/metabolic events will be presented These events will be described in relation to pluripotent adult mesenchymal stem cell proliferation and differentiation as being representative of the relationship applicable to the process in all stem cells.

Mammalian cell intermediary energy metabolism is comprised of a collection of several major metabolic pathways that involve carbohydrate, lipid, and protein metabolism, and terminal oxidation pathways. At some point in mammalian cell aerobic metabolism, essentially all of the pathways converge on and/or arise from the Krebs cycle (also called the citric acid cycle or the tricarboxyllic acid cycle) (Figure 2). In the typical Krebs cycle that generally operates in most cells (Figures 3 and 4 A), the source of citrate is derived from its synthesis in the mitochondria by the condensation of acetylCoA (acetyl coenzyme A) and OAA (oxalacetate). Citrate enters the oxidative phase of the Krebs cycle by its conversion to isocitrate via the $m$-aconitase reaction. Downstream reactions oxidize isocitrate to $O A A$; which then condenses with another molecule of acetylCoA, and another Krebs cycle event occurs.

Consequently, the oxidation of citrate via the Krebs cycle involves the loss of two carbons as $\mathrm{CO}_{2}$ and the regeneration of the four carbon OAA. In typical normal cell metabolism, the acetylCoA is produced from pyruvate, which is generated from the glycolytic utilization of glucose. 

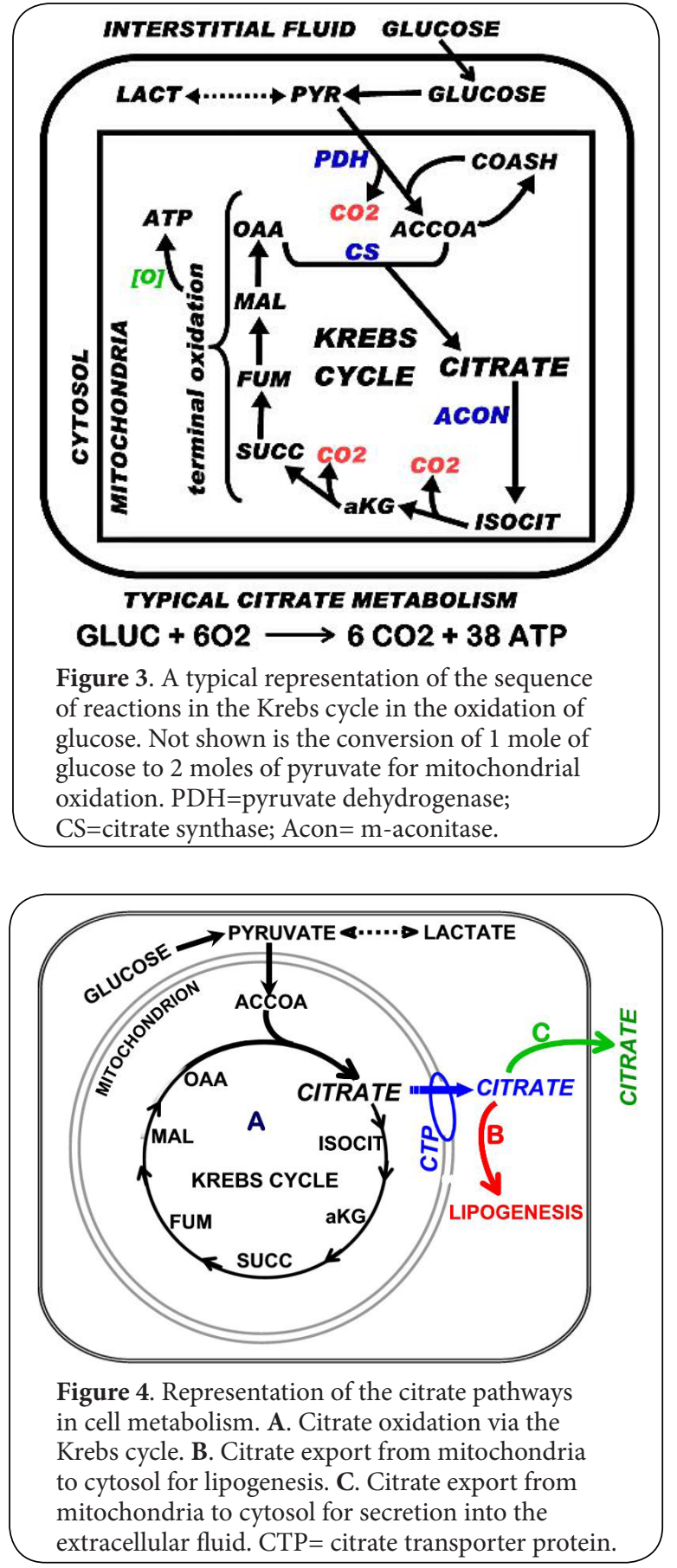

This is the major metabolic pathway of ATP production in which the complete oxidation of 1 mole of glucose can produce 38 moles of ATP [note: this is the theoretical optimal ATP production]. Citrate oxidation via the Krebs cycle produces 24 of the 38 moles of ATP from each mole of glucose. Consequently, the cellular citrate is synthesized in the mitochondria, where it is then utilized via its entry into the Krebs cycle, especially for ATP production.

However, there exist conditions and cellular requirements in which the above typical role of citrate is altered to meet

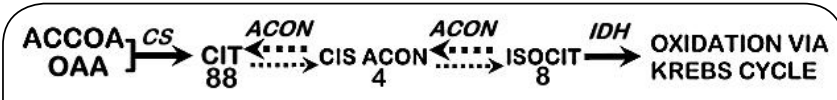

Figure 5. The equilibrium reaction of $\mathrm{m}$-aconitase in typical mammalian cells. CS=citrate synthase;

IDH=isocitrate dehydrogenase; Acon= $\mathrm{m}$-aconitase;

$\mathrm{Cit}=$ citrate; $\mathrm{Cis}$ acon= cis-aconitate; Isocit=isocitrate;

AccoA $=$ acetylCoA; $\mathrm{OAA}=$ oxalacetate.

special functions and activities of the cell (Figure 4). In addition to its utilization via the Krebs cycle, citrate can be transported from the mitochondria to the cytosol where it can be utilized for the metabolic pathway of de novo lipid biosynthesis (i.e., lipogenesis and cholesterogenesis) (Figure 4B). In order for citrate to exit the mitochondria, the upregulation of the mitochondrial citrate transport protein, CTP (Scl25A1) is required (Figure 4). Another alternative that occurs in some specialized cells is the prevention of citrate utilization, so that the synthesized citrate is removed from the cell such as for citrate secretion into the extracellular fluid (Figure 4C). These major roles of citrate are represented in the process of proliferation and differentiation of stem cells.

The above description demonstrates that citrate occupies a central position in cellular metabolism. This is achieved largely by the kinetic properties of the $\mathrm{m}$-aconitase reaction in mammalian cells. In typical mammalian cell metabolism, $\mathrm{m}$-aconitase activity exists in excess relative to other enzymes in the Krebs cycle. This results in the rapid establishment of the $\mathrm{m}$-aconitase equilibrium in which citrate, cis-aconitate, and isocitrate exist in the ratio of $\sim 88: 4: 8$ (Figure 5). This equilibrium is maintained independent of the cellular concentration of citrate; so that most cells exhibit a citrate/isocitrate ratio $\sim 10 / 1$. This relationship limits the conversion of citrate to isocitrate and its subsequent oxidation; so that a significant pool of citrate is maintained and available for any of the pathways shown in (Figure 4).

Representation of stem cell differentiation and citrate metabolic alterations: osteogenic differentiation of mesenchymal stem cells to functional osteoblasts

A stem cell process model is helpful to describe representative citrate-related metabolic alterations in association with the stem cell differentiation process leading to specialized functional cell types. We must acknowledge that cellular metabolic pathways and alterations related to stem cells and their differentiation have received little attention and remain poorly identified. Therefore, this discussion will rely on expected relationships that need to be explored and resolved.

For this presentation we elect to describe the osteogenic differentiation of adult mesenchymal stem cells in the context of production of functional citrate-producing osteoblast in bone formation. It is plausible that this model will involve metabolic transformations that represent the 


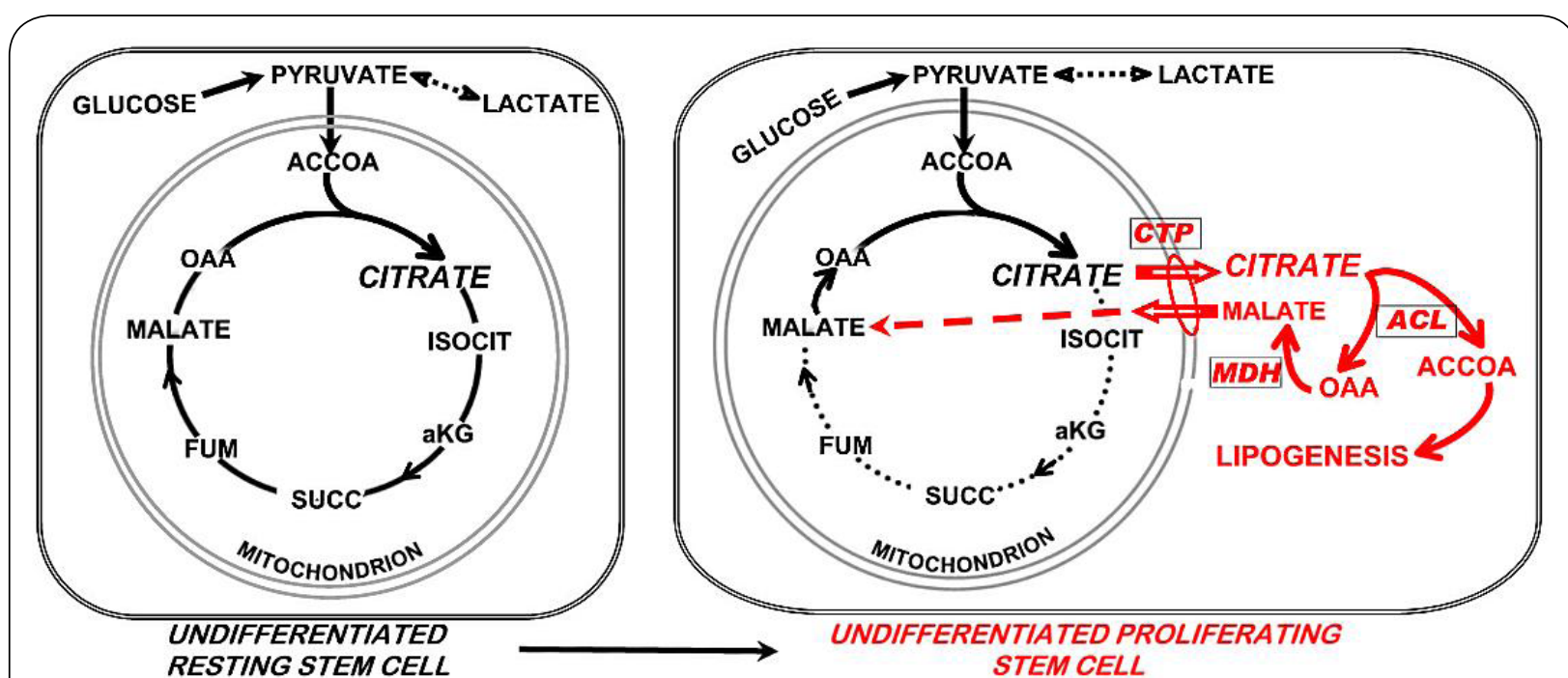

Figure 6. The utilization of citrate for de novo lipogenesis in the genetic/metabolic transformation of highly proliferating undifferentiated stem cells. $\mathrm{CTP}=$ citrate transporter protein; $\mathrm{ACL}=\mathrm{ATP}$-citrate lyase; $\mathrm{MDH}=$ malic dehydrogenase.

three roles of citrate described above (Figure 4).

\section{Genetic/metabolic transformation: undifferentiated} stem cell proliferation

The process will begin with the presence of resident undifferentiated mesenchyme stem cells that exist in a "resting" state. The status of the intermediary energy metabolism of these cells is not established. Komarova et al., [3] using primary rat calvaria culture reported that the differentiation of immature osteoblasts to mineralizing osteoblasts was associated with a transition from predominantly glycolytic utilization of glucose to increased oxidative metabolism. Additional studies purport to show that hypoxia and glycolysis support the proliferation of the undifferentiated stem cells [4-9]; but the involvement of the metabolic requirements (such as de novo lipogenesis) in support of proliferation has not been identified.

Presumably, resting (i.e., non-proliferating) undifferentiated stem cells have no apparent need for de novo lipogenesis or for citrate secretion. Therefore it is most likely that these cells are maintained by glycolysis and, with sufficient aerobic conditions, the typical operation of the Krebs cycle in which citrate is oxidized (Figures 3 and 4A). At some point, a requirement for bone formation occurs, and these undifferentiated resting cells are recruited in preparation for initiation of differentiation. This requires an increase in the population of the mesenchymal stem cells for their differentiation to osteoblasts. To achieve this, the resting cell metabolism must be directed to provide de novo lipogenesis/cholesterogenesis: which is a requirement for membraneogenesis of highly proliferating cells.

This involves a "genetic/metabolic" transformation as shown in (Figure 6). De novo lipogenesis requires a cytosolic source of acetylCoA, which is the building block for lipid biosynthesis. This source of acetylCoA is derived from the mitochondrial synthesized citrate, which is transported to the cytosol. For this to occur, the mitochondrial citrate transporter (CTP) must be upregulated. In the cytosol, ATP-citrate lyase activity must also be upregulated, which converts citrate to oxalacetate+acetylCoA. The acetylCoA then enters the downstream lipogenic/cholesterogenic pathway required for cell proliferation. Since the loss of citrate from its entry and oxidation by the Krebs cycle removes the regeneration of OAA, a new source of OAA is required to permit further synthesis of citrate. This is obtained from the cytosolic OAA, which is oxidized to malate by cytosolic malate dehydrogenase (c-MDH); and the malate is imported into the mitochondria as the counter ion for the CTP export of citrate. Consequently, the resting stem cells must undergo the genetic/metabolic transformation involving upregulation of CTP, ATP-CL, and $\mathrm{c}-\mathrm{MDH}$ to provide the initiating events for accelerated de novo lipogenesis to increase their population in preparation for differentiation. We must emphasize that this discussion deals with the role of citrate. There are other related genetic/metabolic changes that accompany those that we have described.

It becomes apparent that glucose utilization via glycolysis that results in the stoichiometric production of lactate will not provide the synthesis of citrate required for cytosolic acetylCoA for the proliferating stem cells. Another important consideration is that the production of one mole of palmitate+lanosterol (the precursors to lipogenic/cholesterogenic synthesis) requires $~ 70 \%$ of the ATP production from glucose via citrate synthesis. Under such conditions, the highly proliferating stem cells might incorporate alternative potential sources of 


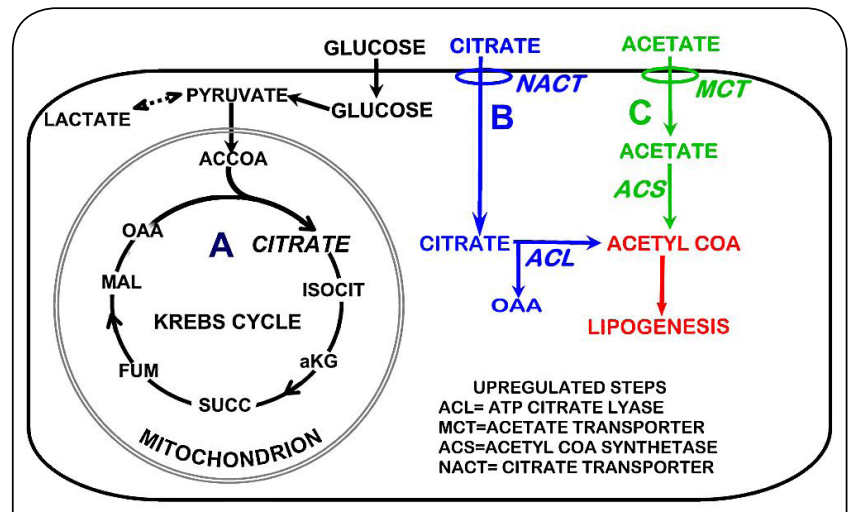

Figure 7. Sparing effect on citrate oxidation by possible alternative sources of cytosolic acetylCoA for de novo lipogenesis in highly proliferating stem cells.

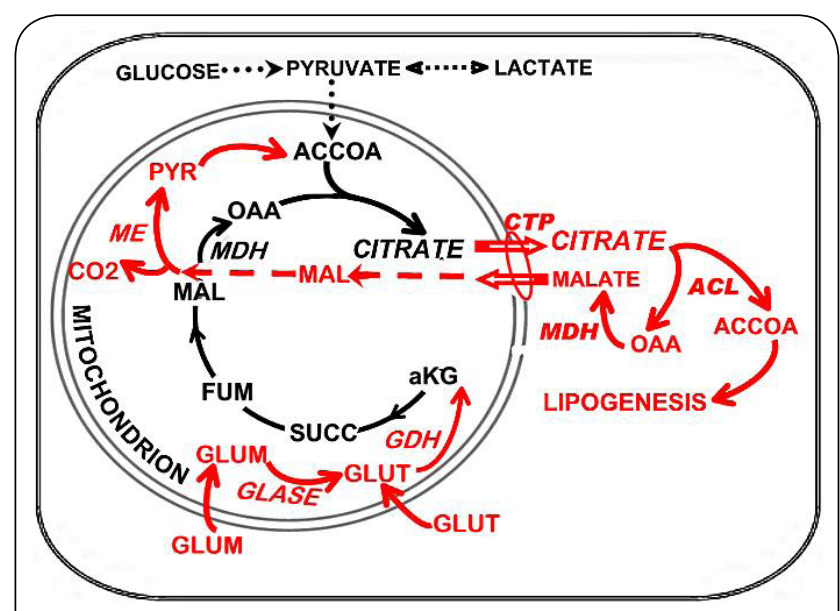

Figure 8. A glutamine/glutamate pathway for citrate synthesis for de novo lipogenesis in highly proliferating stem cells. $\mathrm{ME}=$ malic enzyme; $\mathrm{MDH}=$ malic dehydrogenase; GLASE=glutaminase; $\mathrm{GDH}=$ glutamic dehydrogenase; $\mathrm{ACL}=\mathrm{ATP}$-citrate lyase; $\mathrm{CTP}=$ citrate transport protein.

cytosolic acetylCoA in order to spare the mitochondrial citrate utilization via the Krebs cycle for bioenergetic and synthetic requirements.

The sparing of citrate for Krebs cycle oxidation and ATP production could be achieved by the utilization of plasma citrate (Figure 7B) as the source of cytosolic acetylCoA. Generally in mammalian cells, plasma citrate is not a source of cellular citrate. The plasma concentration of citrate is $\sim 150 \mathrm{uM}$ and does not permeate the plasma membrane. However, under special conditions and requirements, cellular uptake of citrate can be achieved by expression of the $\mathrm{Na}$ coupled citrate transporter such as NaCT (SLC13A5) [11-13].

Another alternative (Figure 7C) is plasma acetate ( 100uM), which is known to be taken up by mammalian cells. The transport of acetate into cells generally requires the presence of a plasma membrane monocarboxyllate transporter, MCT(SLC16A) $[14,15]$. In addition, once transported into the cell, the acetate must be preferentially converted to acetylCoA. This requires cytosolic acetylCoA synthetase (ACS; AceCS1), which is not highly expressed in most mammalian cells; and therefore the acetate is not generally utilized for lipogenesis. However, ACS has been shown to be upregulated in proliferating cells such as in embryogenesis and in specialized cells that undergo de novo lipogenesis [16-18]. Therefore the acetate pathway shown in (Figure 7C) might be important in the highly proliferating undifferentiated stem cells.

The pathways described above involve a dependency on glucose in the synthesis of citrate. However, glutaminolysis can result in citrate synthesis for lipogenesis, even in the absence of glycolysis, as has been proposed for tumor cells $[19,20]$. It is noteworthy that Fernandes et al., [10] reported the proliferation of stem cells with glutamine in the absence of glucose availability in the medium. Thus, a glutamine/glutamate pathway for citrate production in concert with lipogenesis [17] as represented in 8 could exist in proliferating stem cells. Glutamine deamination to glutamate or direct cellular uptake transport of glutamate can be oxidized to alpha ketoglutarate in the mitochondria; which would lead to malate production and its oxidation by MDH to OAA. In the presence of malic enzyme, malate from cytosol can be decarboxylated to pyruvate, which is oxidized to acetylCoA. The export of citrate via citrate transport will regenerate malate as the source of pyruvate.

Genetic/metabolic transformation: osteogenic stem cell differentiation to citrate-producing osteoblasts Bone contains extremely high levels of citrate, which has recently been discovered to be an essential component of the apatite nanocrystal [21-23]. Recent information supports the likelihood that the osteoblasts are the source of citrate that is incorporated into bone during bone formation [24]. For this to occur, altered citrate metabolism must occur in the transformation of the undifferentiated mesenchymal stem cells to the functional citrate-producing osteoblasts. An analogous situation exists in prostate epithelial cells, which are specialized citrate-producing cells that secrete high levels of citrate into the prostatic fluid $[25,26]$. About two decades of reported studies resulted in our identification of the pathway of citrate production in those cells, and similar metabolic alterations probably exist in the osteoblasts (Figure 9).

The uniquely high citrate production as exists in prostate and bone requires that most of the citrate that is synthesized is secreted from the cells. This occurs in prostate by the cellular accumulation of high zinc levels, which inhibits $\mathrm{m}$-aconitase activity and prevents citrate oxidation [27]. The increase in zinc accumulation results from the expression of the ZIP1 (SIc39A1) zinc uptake transporter $[26,28]$. It is notable that ZIP1 is upregulated and zinc accumulation is increased as a requirement for the differentiation of human mesenchymal stem cells to 


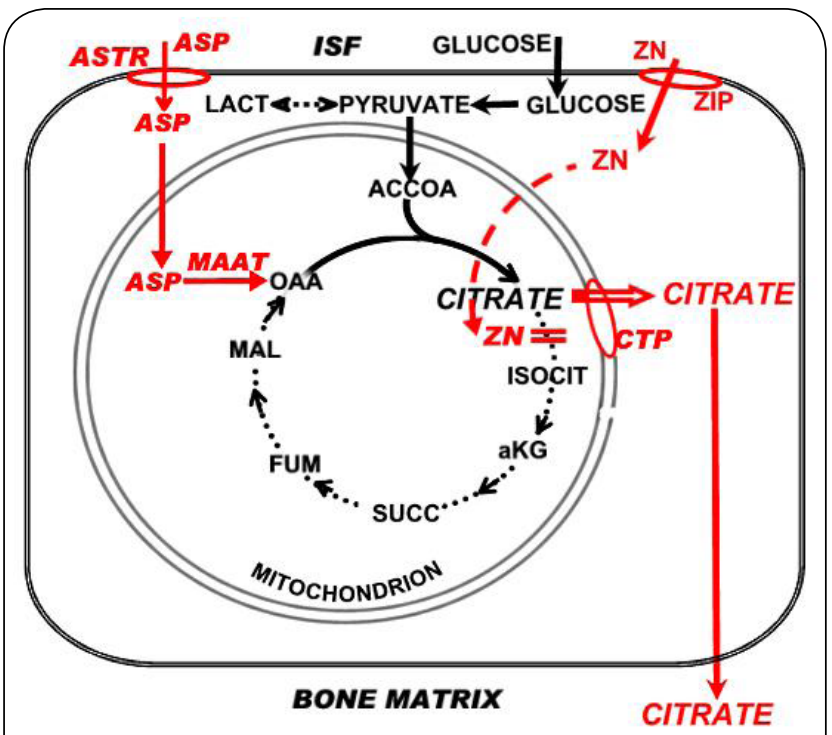

Figure 9. The pathway for citrate production by differentiated osteoblasts for citrate incorporation into bone. Red represents the upregulated events; and the black dashed pathway represents the truncated Krebs cycle. $\mathrm{ASP}=$ aspartate; $\mathrm{ASTR}=$ aspartate transporter; MAAT $=$ mitochondrial aspartate aminotransferase; $\mathrm{CTP}=$ citrate transporter protein; $\mathrm{ZIP}=$ zinc uptake transporter; ISF=interstitial fluid.

mineral-producing osteoblasts [29]. Therefore, this is a likely event for osteoblast citrate production as shown in (Figure 9).

Unlike the other pathways described above, this presents the condition in which a source of OAA as well as acetylCoA is required to provide the six carbons for citrate synthesis. In prostate epithelial cells, the cellular uptake of aspartate from circulation, which is then transaminated by the mitochondrial aspartate aminotransferase reaction (mAAT), provides the source of OAA (Figure 9). Most mammalian cells do not have a requirement for aspartate uptake from circulation since aspartate is a nonessential amino acid that is synthesized by the cells. Therefore, the uptake of aspartate is achieved by the upregulation of an aspartate transporter (such as EAAC1 in prostate cells [30]) as a requirement for the differentiated citrate-producing osteoblasts. Thus, most likely, aspartate is an essential amino acid that is required for the developing osteoblasts.

The removal of citrate from entry into the Krebs cycle and ultimately from the cell has important bioenergetic consequences. As described above, citrate oxidation via the Krebs cycle provides $\sim 67 \%$ of the ATP that is produced by the complete oxidation of glucose; which is lost when the citrate is removed from the metabolic pool. The cells must compensate for this loss, which is achieved by the ATP from increased glucose utilization via glycolysis and increased pyruvate oxidation. This also increases the production of acetylCoA for increased synthesis of citrate. The above events exemplify the widespread implications of altered citrate utilization on the cell's intermediary metabolism.

Genetic/metabolic transformation: citrate implications in stem cell differentiation of other specialized cells In regard to the proliferation of the undifferentiated stem cells, the de novo lipogenesis genetic/metabolic transformation described above (Figures $6,7,8$ ) likely applies to all highly proliferating stem cells. However, the implications and genetic/metabolic alterations involved in the differentiation leading to other specialized cells will vary with the specialized function of the specific cell type. For example, the mesenchymal stem cell adipogenic differentiation to functional adipocytes involves fatty acid synthesis leading to palmitate and triglyceride production. This proceeds via the fatty acid synthesis pathway of lipogenesis as described above. Citrate oxidation via the Krebs cycle is decreased and accumulating citrate is exported to the cytosol where it is converted to acetylCoA for fatty acid synthesis. Consequently the genetic/metabolic events involving citrate metabolism in the differentiation of the stem cell are different for the development of osteoblasts versus adipocytes.

Another example is the differentiation of stem cells to insulin-producing beta cells. Beta cells, like prostate cells and osteoblasts, are also highly specialized zincaccumulating cells; in which the accumulation of high zinc levels is required for the synthesis and secretion of insulin. It is well established that stem cell differentiation to insulin-producing beta cells includes an increased accumulation of zinc [31,32]. However, the zinc uptake transporter(s) and mechanism of zinc accumulation in the beta cells have not been identified; and an important possible expected effect of the high cellular zinc on citrate metabolism (especially inhibition of $\mathrm{m}$-aconitase and citrate oxidation) has not been reported.

\section{The issue of a hypoxic stem cell environment}

The enzyme activity within a cell is dependent upon the abundance of the enzyme and the kinetics of the enzyme. The former is determined by the genetic expression, synthesis, and degradation (i.e., "turnover") of the corresponding "metabolic gene" product. The latter is determined by the localized environment of the cell that alters the kinetics of the enzyme (such as substrate concentration, $\mathrm{pH}$, inhibitors, activators).

This brings attention to the important metabolic issue that arises from the generally accepted view that stem cells often reside in severely hypoxic regions; such as bone marrow MSC existence in a microenvironment of $\sim 1-8 \%$ oxygen [3-9]. In vitro studies [such as 6.8.9] conclude that such hypoxic conditions impose a glycolytic metabolism with stoichiometric production of lactate, which favors the proliferation of stem cells. However, none of the reports recognized, considered, or investigated the metabolic implications in relation to citrate synthesis and 
achieving de novo lipid biosynthesis required for the stem cell proliferation. This is mindful of the "Warburg effect" controversy relating to tumor cell metabolism, which we similarly addressed in recent reviews [2,33]. The idea and conclusion that mammalian cells function, grow, and proliferate under hypoxic in vivo conditions that impose an "anaerobic-like glycolytic metabolism" (even if it is called "accelerated aerobic glycolysis") must be presented in the context of its impact and implications requiring associated metabolic adaptations to permit cellular growth, proliferation and function. It becomes evident that such hypoxic glycolysis prevents the synthesis of citrate and its availability for production of acetylCoA required for de novo lipid biosynthesis in proliferating cells. Under such conditions, the requirement for alternative sources and metabolic pathways for citrate and cytosolic acetylCoA must be recognized, considered, and identified. It has also been suggested that the hypoxic condition that promotes the proliferation of the undifferentiated stem cell is not favorable for their differentiation to specific functional cell types such as osteoblasts and adipocytes; which require increased respiration and aerobic metabolism $[6,8]$. Thus the role and implications of altered intermediary metabolism become paramount events in the process of stem cell proliferation and differentiation.

Native stem cell differentiation vs. experimentalinduced stem cell differentiation: Are the same specialized functional metabolic cells produced?

This question arises when one considers the conditions and factors that are employed in the experimental differentiation of stem cells to specific functional cell types. This is exemplified by the composition of the various differentiation media for directing stem cells to specific cell types. One example is the osteogenic differentiation of human mesenchymal stem cells to functional osteoblasts. Typically, the osteogenic medium is supplemented with dexamethasone, which is a glucocorticoid that promotes gluconeogenesis. Consequently, dexamethasone will inhibit glucose utilization via glycolysis, which will prevent pyruvate production and acetylCoA formation for citrate synthesis. In addition, any available pyruvate, OAA, and malate (such as from protein catabolism amino acids) will be directed toward entry into reversed glycolysis leading to glucose production. Mitochondrial fatty acid oxidation will produce acetylCoA that will react with OAA derived from amino acids to synthesize citrate, which will be oxidized by the Krebs cycle as a source of ATP production in the absence of glucose oxidation. Such gluconeogenic reactions are counter to stem cell differentiation for the function and metabolic capability of osteoblast production of citrate for incorporation into hydoxyapatite in bone formation.

Similarly, adipogenic differentiation media generally include both dexamethasone and insulin. These are essentially physiological opposing agents in which insulin is lipogenic and glycolytic, whereas dexamethasone is lipolytic and gluconeogenic. Insulin promotes glucose utilization leading to citrate synthesis and the utilization of citrate for cytosolic production of acetylCoA for fatty acid production; and dexamethasone promotes fatty acid oxidation and glucose production.

Such seemingly conflicting relationships raise the concern as to the metabolic and physiological characteristics and capabilities of the experimentally differentiated specialized cell types in relation to the corresponding native developed cell type. When this potential disparity is identified, is it not important to establish more appropriate conditions that might promote the differentiation of the stem cells to produce more representative normal metabolic and functional specialized cells?

This issue is again represented in the osteogenic differentiation leading to the development of osteoblasts. Presently, the success of the differentiation of the stem cells in osteogenic medium is determined by the production of mineralized hydoxyapaptite, in the absence of determination and confirmation of citrate production and incorporation of citrate in the mineralized hydroxyapatite structure. The conditions of the osteogenic medium that are generally employed will not likely promote osteoblast citrate production [24]. For example, the media should contain physiological concentrations of zinc, and also aspartate. Most likely, dexamethasone should be excluded and substituted by the addition of vitamin D. Vitamin D promotes bone citrate production; and its deficiency results in rickets characterized by low citrate in the bone [34-36]. Moreover, vitamin D has been shown to be a satisfactory supplement, in the absence of dexamathasone, for the promotion of the stem cell osteogenesis [37]. Thus, under the conditions currently employed for osteogenesis, the product of the experimentally differentiated osteoblast will be the formation of bone that does not contain the required composition of citrate. Since citrate is essential for providing the bone structure that exhibits the important properties of stability, strength, and resistance to fracture; in its absence, the osteoblasts are incapable of normal bone formation.

\section{Conclusions}

Genetic/metabolic alterations in intermediary energy metabolism are essential events in the proliferation and differentiation of stem cells. However, relatively little information exists regarding stem cell intermediary metabolism during proliferation and differentiation. This especially applies to the central role of citrate metabolism in the highly proliferating undifferentiated stem cells and in their differentiation to specific functional cell types. This presentation has provided representative examples and identification of some relevant genetic and metabolic alterations and implications in the proliferation/ differentiation process. Such information is essential for 
the determination of the important factors and conditions associated with and required for the development and characterization of normal functional metabolic cell types. It then become essential that the conditions and outcome of experimental differentiation of stem cells is the development of specialized cells that represent the functional and metabolic activities of the native specialized cells. This is especially important for the success of stem cell therapy for treatment of many pathological conditions. Consequently, the relationships of intermediary metabolism in stem cells and the differentiation process is a critical area that requires more recognition, focus, and research support. In the absence of such information, the important factors and events associated with stem cell differentiation cannot be fully identified and understood.

\section{Competing interests}

The authors declare that they have no competing interests.

Authors' contributions

Both LCC and RBF contributed to the development and writing of this review article. The final manuscript was read and approved by both authors.

\section{Acknowledgement}

The studies of the authors (LCC and RBF)

described in this review were supported by $\mathrm{NIH}$

grants CA79903, CA93443, and DK42839.

Publication history

Received: 12-Apr-2013 Revised: 08-May-2013

Accepted: 09-May-2013 Published: 11-May-2013

\section{References}

1. Costello LC and Franklin RB: Integration of molecular genetics and proteomics with cell metabolism: how to proceed; how not to proceed! Gene 2011, 486:88-93. | Article | PubMed

2. Costello LC and Franklin RB: The genetic/metabolic transformation concept of carcinogenesis. Cancer Metastasis Rev 2012, 31:123-30. | Article I PubMed

3. Komarova SV, Ataullakhanov FI and Globus RK: Bioenergetics and mitochondrial transmembrane potential during differentiation of cultured osteoblasts. Am J Physiol Cell Physiol 2000, 279:C1220-9. I Article I PubMed

4. Kim JM, Jeong D, Kang HK, Jung SY, Kang SS and Min BM: Osteoclast precursors display dynamic metabolic shifts toward accelerated glucose metabolism at an early stage of RANKL-stimulated osteoclast differentiation. Cell Physiol Biochem 2007, 20:935-46. | Article I PubMed

5. Teitelbaum SL and Ross FP: Genetic regulation of osteoclast development and function. Nat Rev Genet 2003, 4:638-49. | Article I PubMed

6. Pattappa G, Heywood HK, de Bruijn JD and Lee DA: The metabolism of human mesenchymal stem cells during proliferation and differentiation. J Cell Physiol 2011, 226:2562-70. | Article | PubMed

7. Mischen BT, Follmar KE, Moyer KE, Buehrer B, Olbrich KC, Levin LS, Klitzman B and Erdmann D: Metabolic and functional characterization of human adipose-derived stem cells in tissue engineering. Plast Reconstr Surg 2008, 122:725-38. | Article | PubMed

8. Malladi P, Xu Y, Chiou M, Giaccia AJ and Longaker MT: Effect of reduced oxygen tension on chondrogenesis and osteogenesis in adipose-derived mesenchymal cells. Am J Physiol Cell Physiol 2006, 290:C1139-46. | Article | PubMed
9. Grayson WL, Zhao F, Bunnell B and Ma T: Hypoxia enhances proliferation and tissue formation of human mesenchymal stem cells. Biochem Biophys Res Commun 2007, 358:948-53. | Article | PubMed

10. Fernandes TG, Fernandes-Platzgummer AM, da Silva CL, Diogo MM and Cabral JM: Kinetic and metabolic analysis of mouse embryonic stem cell expansion under serum-free conditions. Biotechnol Lett 2010, 32:171-9. | Article | PubMed

11. Bergeron MJ, Clemencon B, Hediger MA and Markovich D: SLC13 family of $\mathrm{Na}(+)$-coupled di- and tri-carboxylate/sulfate transporters. Mol Aspects Med 2013, 34:299-312. | Article | PubMed

12. Gopal E, Miyauchi S, Martin PM, Ananth S, Srinivas SR, Smith SB, Prasad PD and Ganapathy V: Expression and functional features of $\mathrm{NaCT}$, a sodium-coupled citrate transporter, in human and rat livers and cell lines. Am J Physiol Gastrointest Liver Physiol 2007, 292:G4028. | Article | PubMed

13. Weerachayaphorn J and Pajor AM: Identification of transport pathways for citric acid cycle intermediates in the human colon carcinoma cell line, Caco-2. Biochim Biophys Acta 2008, 1778:1051-9. | Article | PubMed Abstract | PubMed Full Text

14. Takebe K, Nio-Kobayashi J, Takahashi-Iwanaga H, Yajima T and Iwanaga T: Cellular expression of a monocarboxylate transporter (MCT1) in the mammary gland and sebaceous gland of mice. Histochem Cell Biol 2009, 131:401-9. | Article | PubMed

15. Moschen I, Broer A, Galic S, Lang F and Broer S: Significance of short chain fatty acid transport by members of the monocarboxylate transporter family (MCT). Neurochem Res 2012, 37:2562-8. | Article I PubMed

16. Loikkanen I, Haghighi S, Vainio S and Pajunen A: Expression of cytosolic acetyl-CoA synthetase gene is developmentally regulated. Mech Dev 2002, 115:139-41. | Article | PubMed

17. Oikawa E, lijima $H$, Suzuki T, Sasano $H$, Sato $H$, Kamataki A, Nagura $H$, Kang MJ, Fujino T, Suzuki $H$ and Yamamoto TT: A novel acylCOA synthetase, ACS5, expressed in intestinal epithelial cells and proliferating preadipocytes. J Biochem 1998, 124:679-85. | Article | PubMed

18. Sone $H$, Shimano $H$, Sakakura $Y$, Inoue $N$, Amemiya-Kudo $M$, Yahagi N, Osawa M, Suzuki H, Yokoo T, Takahashi A, lida K, Toyoshima H, Iwama A and Yamada N: Acetyl-coenzyme A synthetase is a lipogenic enzyme controlled by SREBP-1 and energy status. Am J Physiol Endocrinol Metab 2002, 282:E222-30. | Article | PubMed

19. Moreadith RW and Lehninger AL: The pathways of glutamate and glutamine oxidation by tumor cell mitochondria. Role of mitochondrial NAD(P)+-dependent malic enzyme. J Biol Chem 1984, 259:6215-21. | Article | PubMed

20. McKeehan WL: Glycolysis, glutaminolysis and cell proliferation. Cell Biol Int Rep 1982, 6:635-50. | Article | PubMed

21. Hu YY, Rawal A and Schmidt-Rohr K: Strongly bound citrate stabilizes the apatite nanocrystals in bone. Proc Natl Acad Sci U S A 2010, 107:22425-9. | Article | PubMed Abstract | PubMed Full Text

22. Hu Y-Y, Liu XP, Ma X, Rawal A, Prozorov T, Akinc M, Mallapragada SK, Schmidt-Rohr K: Biomimetic self-assembling copolymer_ hydroxyapatite nanocomposites with the nanocrystal size controlled by citrate. Chem Mater 2011, 23: 2481-2490. | Article

23. Schmidt-Rohr K: Citrate key in bone's nanostructure. Science Daily (June 8, 2011). I Website

24. Costello LC, Franklin RB, Chellaiah M, Reynolds MA: The important role of osteoblasts and citrate production in bone formation: "osteoblast citration" as a new concept for an old relationship. The Open Bone J 2012, 4:27-34. I Pdf

25. Costello LC, Franklin RB and Feng P: Mitochondrial function, zinc, and intermediary metabolism relationships in normal prostate and prostate cancer. Mitochondrion 2005, 5:143-53. | Article | PubMed

26. Costello LC and Franklin RB: The clinical relevance of the metabolism of prostate cancer; zinc and tumor suppression: connecting the dots. Mol Cancer 2006, 5:17. | Article | PubMed Abstract | PubMed Full Text 
27. Costello LC, Liu Y, Franklin RB and Kennedy MC: Zinc inhibition of mitochondrial aconitase and its importance in citrate metabolism of prostate epithelial cells. J Biol Chem 1997, 272:28875-81. | Article | PubMed

28. Franklin RB, Ma J, Zou J, Guan Z, Kukoyi BI, Feng P and Costello LC: Human ZIP1 is a major zinc uptake transporter for the accumulation of zinc in prostate cells. J Inorg Biochem 2003, 96:435-42. | Article | PubMed

29. Tang Z, Sahu SN, Khadeer MA, Bai G, Franklin RB and Gupta A: Overexpression of the ZIP1 zinc transporter induces an osteogenic phenotype in mesenchymal stem cells. Bone 2006, 38:181-98. | Article I PubMed

30. Franklin RB, Zou J, Yu Z and Costello LC: EAAC1 is expressed in rat and human prostate epithelial cells; functions as a highaffinity L-aspartate transporter; and is regulated by prolactin and testosterone. BMC Biochem 2006, 7:10. | Article | PubMed Abstract | PubMed Full Text

31. Shiroi A, Yoshikawa M, Yokota $H$, Fukui $H$, Ishizaka S, Tatsumi $K$ and Takahashi Y: Identification of insulin-producing cells derived from embryonic stem cells by zinc-chelating dithizone. Stem Cells 2002, 20:284-92. | Article | PubMed

32. Baharvand H, Jafary H, Massumi M and Ashtiani SK: Generation of insulin-secreting cells from human embryonic stem cells. Dev Growth Differ 2006, 48:323-32. | Article | PubMed

33. Costello LC and Franklin RB: 'Why do tumour cells glycolyse?': from glycolysis through citrate to lipogenesis. Mol Cell Biochem 2005, 280:1-8. | Article | PubMed

34. Dixon TF and Perkins HR: Citric acid and bone metabolism. Biochem $J$ 1952, 52:260-5. | Article | PubMed Abstract | PubMed Full Text

35. Steenbock $H$ and Bellin SA: Vitamin D and tissue citrate. J Biol Chem 1953, 205:985-91. | Article | PubMed

36. Carlsson A and Hollunger G: The effect of vitamin D on the citric acid metabolism. Acta Physiol Scand 1954, 31:317-33. | Article | PubMed

37. Zhou YS, Liu YS and Tan JG: Is 1, 25-dihydroxyvitamin D3 an ideal substitute for dexamethasone for inducing osteogenic differentiation of human adipose tissue-derived stromal cells in vitro? Chin Med J (Engl) 2006, 119:1278-86. | Article | PubMed

\section{Citation:}

Costello LC and Franklin RB: A review of the important central role of altered citrate metabolism during the process of stem cell differentiation. journal of Regenerative Medicine and Tissue Engineering 2013, 2:1. http://dx.doi.org/10.7243/2050-1218-2-1 